\title{
Controlling DNA Bundle Size and Spatial Arrangement in Self- assembled Arrays on Superhydrophobic Surface
}

\author{
Gabriele Ciasca - Massimiliano Papi - Valentina Palmieri $\cdot$ Michela Chiarpotto $\cdot$ Simone Di Claudio • \\ Adele De Ninno • Ennio Giovine - Gaetano Campi - Annamaria Gerardino • Luca Businaro • Marco De Spirito
}

Received: 29 September 2014/ Accepted: 28 November 2014/Published online: 30 December 2014

(C) The Author(s) 2014. This article is published with open access at Springerlink.com

\begin{abstract}
The use of superhydrophobic surfaces (SHSs) is now emerging as an attractive platform for the realization of one-dimensional (1D) nanostructures with potential applications in many nanotechnological and biotechnological fields. To this purpose, a strict control of the nanostructures size and their spatial arrangement is highly required. However, these parameters may be strongly dependent on the complex evaporation dynamics of the sessile droplet on the SHS. In this work, we investigated the effect of the evaporation dynamics on the size and the spatial arrangement of self-assembled 1D DNA bundles. Our results reveal that different arrangements and bundle size distributions may occur depending on droplet evaporation stage. These results contribute to elucidate the formation mechanism of 1D nanostructures on SHSs.
\end{abstract}

Keywords Superhydrophobicity $\cdot$ DNA array $\cdot$ Cassie state $\cdot$ Wenzel state $\cdot$ 1D nanostructures $\cdot$ Self-assembly

\section{Introduction}

One-dimensional (1D) nanostructures such as polymeric, small molecules and inorganic types are currently under much investigation for their unique mechanical, optical, and electronic properties, as well as possible applications in design and realization of novel biomedical devices [1]. Over the past 20 years, most of the research efforts have been devoted to the synthesis of 1D nanostructures. To this

Luca Businaro and Marco De Spirito share equal credit for senior authorship.

G. Ciasca · M. Papi (ه) - V. Palmieri - M. Chiarpotto ·

S. Di Claudio · M. De Spirito

Istituto di Fisica, Universitá Cattolica SC, L.go Francesco Vito

1, 00168 Rome, Italy

e-mail: m.papi@rm.unicatt.it

A. De Ninno - E. Giovine - A. Gerardino - L. Businaro Istituto di Fotonica e Nanotecnologie-CNR, Via Cineto Romano 42, 00156 Rome, Italy

G. Campi

Institute of Crystallography-CNR, Via Salaria Km 29, 0016 Monterotondo, Rome, Italy purpose, many strategies based on top-down or bottom-up approaches have been successfully developed and applied [2]. Aside from the establishment of an effective synthesis route, the integration of $1 \mathrm{D}$ nanostructures into functional devices requires development of novel strategies to align such nanostructures in a parallel, scalable, and highly reproducible manner. A possible approach is based on the use of standard top-down lithographic techniques such as electron-beam lithography. However, this approach is often challenging, expensive, and time-consuming [1-4].

These limitations can be overcome by using superhydrophobic patterned surface. Historically, superhydrophobic surfaces have attracted much attention because of their self-cleaning properties that make them suitable for a variety of technological and industrial applications [5-15]. However, since a few years back, researchers have moved their interest to investigate whether superhydrophobic surfaces could be actively exploited to manipulate matter at the nanoscale level. This research effort led to design and test new devices with unexplored and attractive properties. Recently, the pioneering work of $\mathrm{Su}$ and co-workers demonstrated the possibility to exploit superhydrophobic surfaces to induce the self-assembly of strictly aligned organic filaments [16]. This result stimulated an intense 
research effort toward the realization of high-aspect ratio one-dimensional nanostructures with possible application in a variety of fields, including plasmonic materials (gold and silver nanoparticles), catalytic compounds, and DNA filaments [17-23].

The mechanism of the deposition of aligned and suspended filaments was described very recently for DNA bundles [19]. Briefly, a droplet containing DNA can be deposited onto a superhydrophobic surface wetted in the Cassie state. Under evaporation conditions, the retracting drop edge stretches DNA filaments along the de-wetting direction. While receding, the droplet forms capillaries which pin to the pillar protrusions allowing for the precise control of bundles position and orientation. For clarity, this mechanism is summarized in Fig. 1.

This self-assembling mechanism has the advantage of producing a remarkably uniform nanostructure size distribution under specific conditions. This feature is very important for device applications because well-aligned and uniform 1D nanostructures exhibit superior properties that have not been found in those of disordered arrangements [1]. However, the experimental conditions for this uniform size distribution have not been fully explored.

In order to explore these conditions, we investigated the size distribution and the spatial arrangement of selfassembled 1D DNA bundles obtained by droplet evaporation on SHSs. We revealed the presence of three spatial regions encompassing three different DNA bundle arrangements with different size distributions.

\section{Materials and Methods}

DNA isolation was performed according to standard phenol/chloroform protocol as described elsewhere [19]. Superhydrophobic surfaces were fabricated directly on silicon substrate by electron-beam lithography (Vistec EPBG-5HR acceleration voltage: $100 \mathrm{keV}$ ) and inductively coupled plasma (ICP) Si etching [22-25]. A 400-nm-thick layer PMMA $950 \mathrm{~K} 9 \%$ was spun on a $\mathrm{Si}$

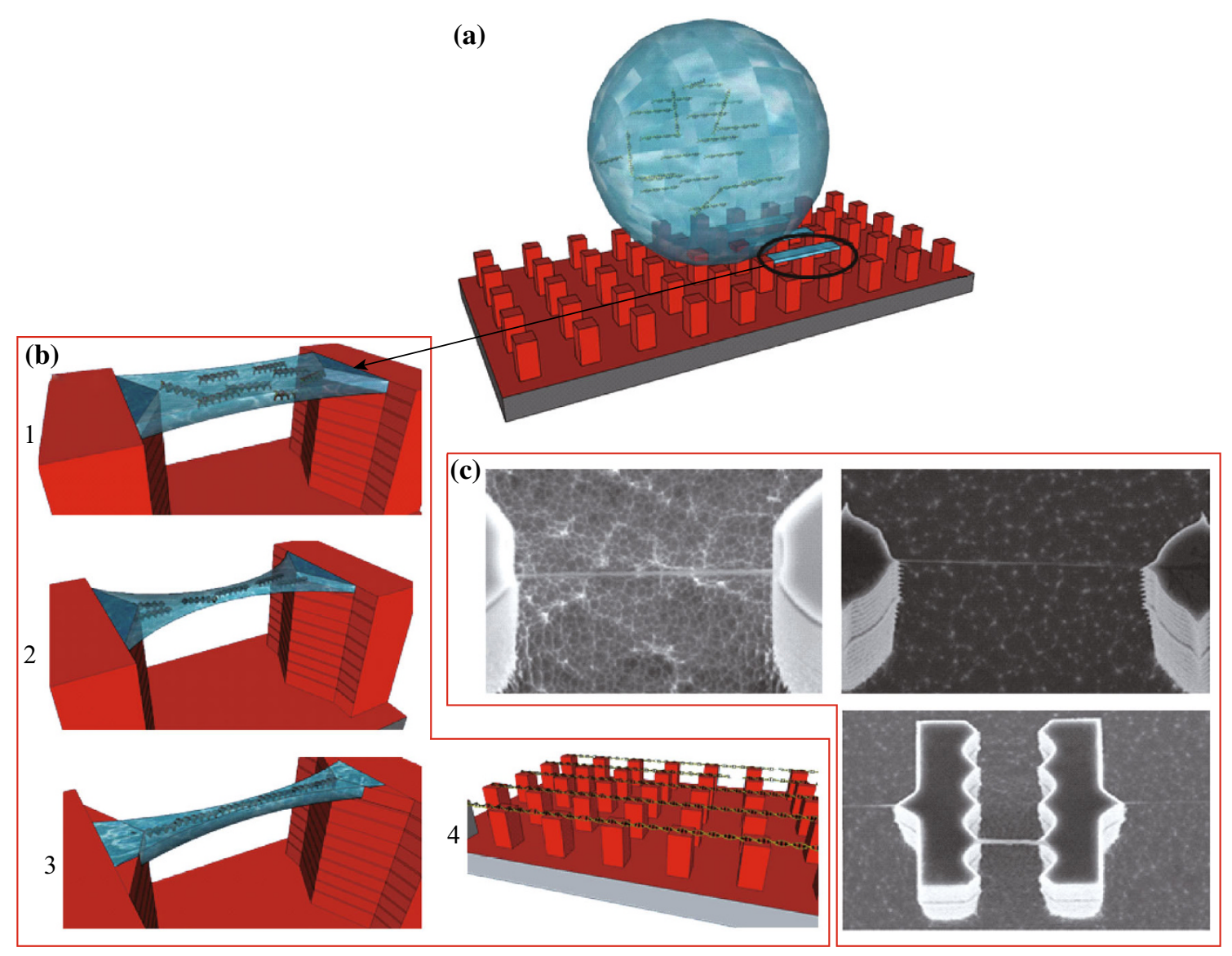

Fig. 1 a Schematic view of the typical pillar-based superhydrophobic patterned surfaces, where a droplet containing DNA has been deposited. b Water capillaries between adjacent pillars (step 1); Water capillary evaporation pushes together the DNA strands (step 2); Formation of stable DNA bundles on the tips side (step 3); Highly ordered array of aligned 1D filaments (step 4). c SEM image of stretched DNA bundles on three different pillar geometries 


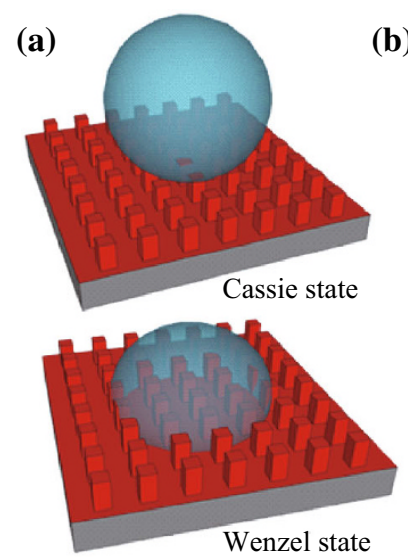

(f)

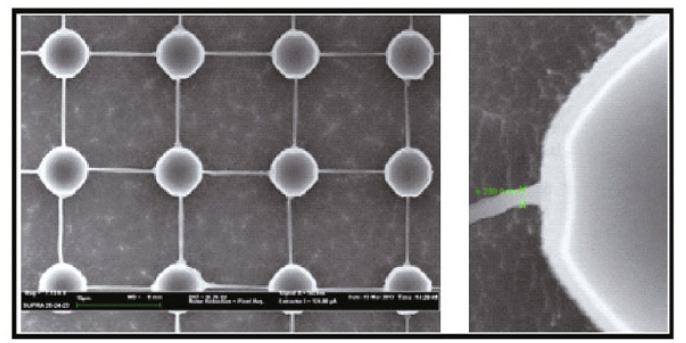

(b)
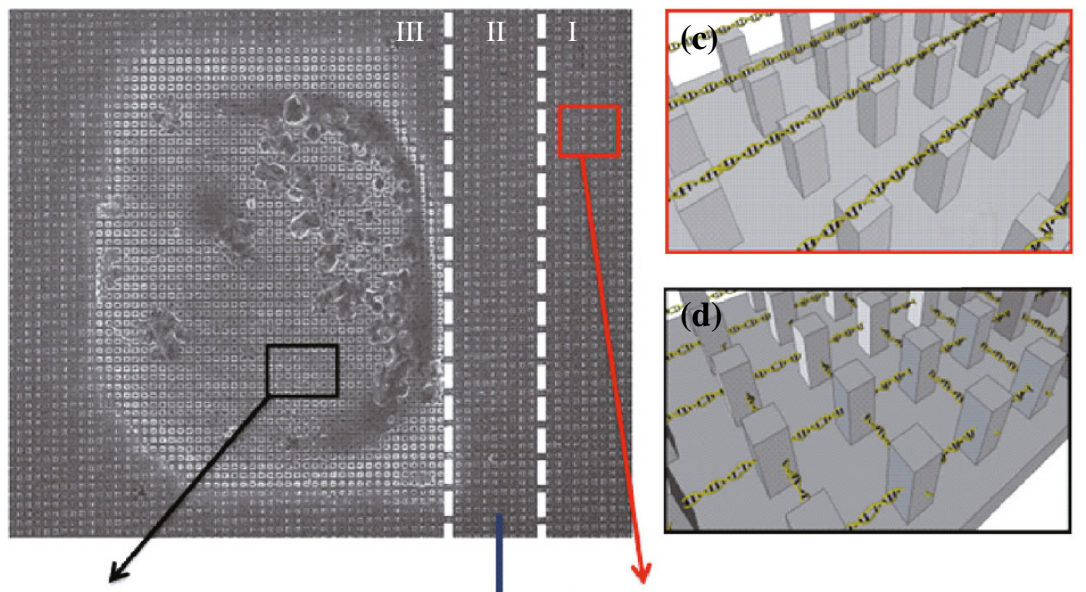

(e)

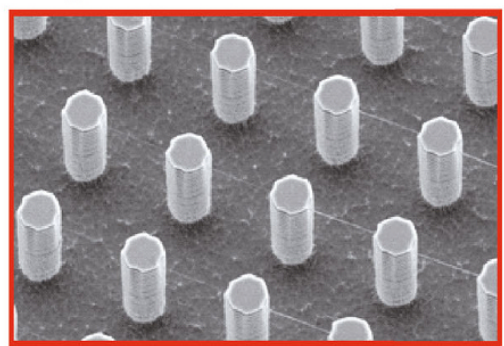

Fig. 2 a Schematic view of a water droplet on a superhydrophobic surface wetted in the Cassie state (above) and in the Wenzel state (below). b Typical DNA stain on a superhydrophobic surface due to the Cassie-to-Wenzel transition. c Schematic view of the typical DNA bundle arrangement occurring in region I; d Schematic view of the DNA bundle array formed in region III. e SEM image of the typical DNA arrangement in region I; f SEM image of the peculiar DNA arrangement that might be obtained inside the DNA stain in particular experimental conditions [20]
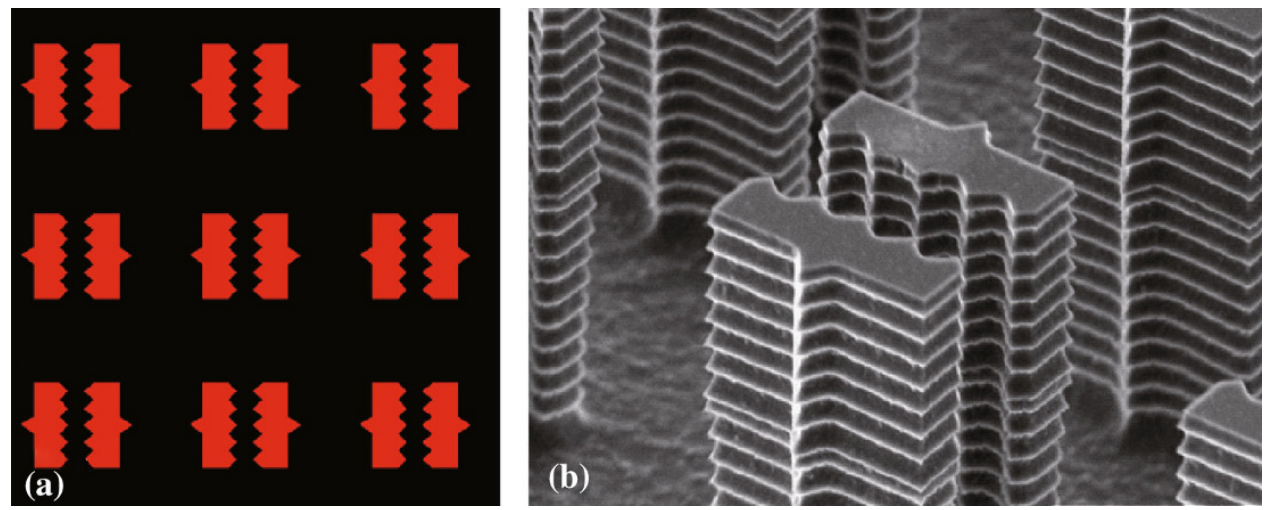

Fig. 3 a Schematic view of adopted superhydrophobic surface; b Tilted SEM image of the microstructured superhydrophobic surface

wafer, exposed with a dose of $700 \mu \mathrm{C} \mathrm{cm}{ }^{-2}$, and developed. A 30-nm-thick Cr film was then deposited by e-gunassisted evaporation and lifted off. The device pattern was transferred on the substrate by ICP etching. After cleaning in Piranha solution $\left(\mathrm{H}_{2} \mathrm{SO}_{4} / \mathrm{H}_{2} \mathrm{O}_{2}=3: 1\right)$, both microstructured $\mathrm{Si}$ wafers were silanized with $10 \%$ trimethylchlorosilane in toluene to impart the superhydrophobic behavior. This fabrication step was detailed discussed in Ref. [26].

Self-assembled arrays of DNA bundles were obtained by deposition of $5 \mu \mathrm{L}$ droplets containing DNA at different concentrations. After deposition, the superhydrophobic surface was tilted by $10^{\circ}$ and the droplet was let to completely evaporate at room temperature. In this configuration, the sample solution droplet slowly slips downward along the tilted surface, allowing for the formation of highly ordered arrays of aligned 1D DNA filaments. The superhydrophobic surfaces were characterized by scanning electron microscopy (SEM, Zeiss Supra 120) after deposition of a 6-nm-thin Cr layer in order to avoid charging effects and to protect DNA filaments from e-beam damage [27, 28]. 

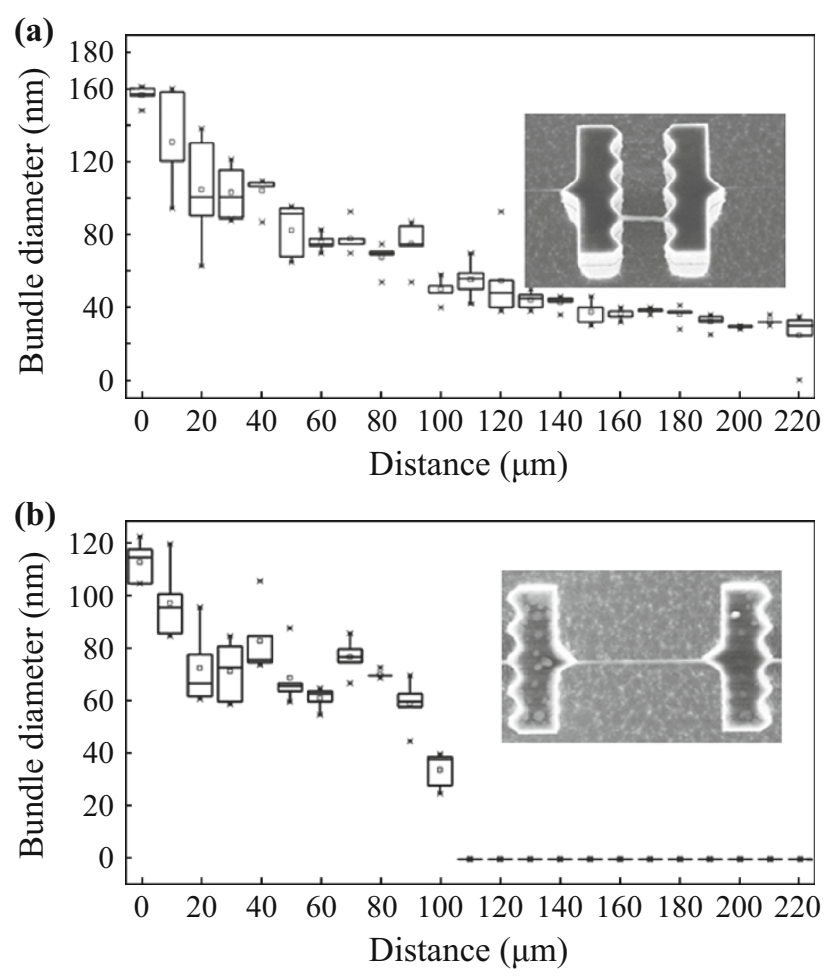

Fig. 4 a The average diameter of the bundles pinned inside the sawshaped pillars in region II as a function of the distance from the DNA stain shown in Fig. 2b. b The average diameter of the bundles pinned outside the saw-shaped pillars as a function of the distance from the stain. A drop volume of $5 \mu \mathrm{L}$ and a DNA concentration of $150 \mathrm{ng} \mu \mathrm{L}^{-1}$ was used

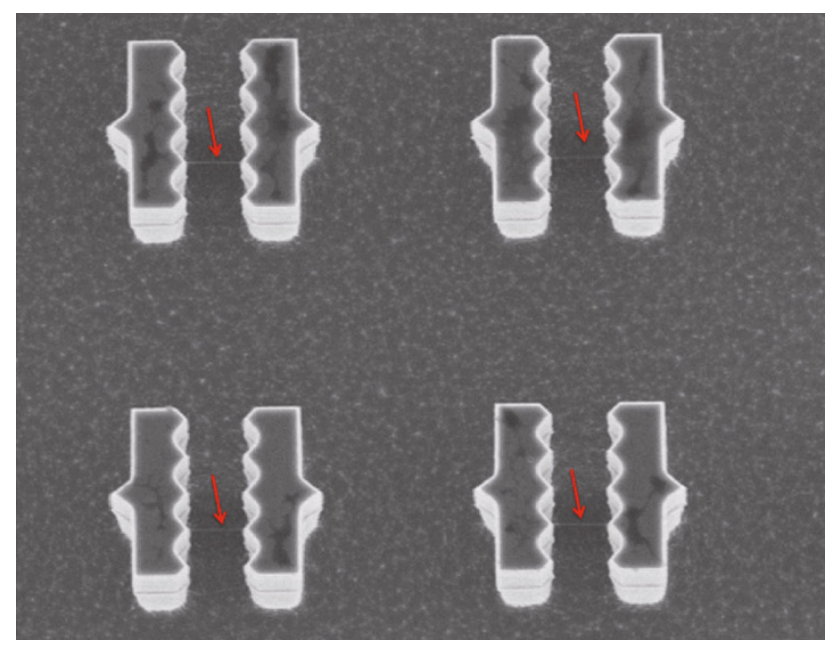

Fig. 5 SEM images of DNA far from the stain where DNA bundles pinned outside the saw-shaped pillars (undetectable) and inside the saw-shaped pillars (detectable)

\section{Results and Discussion}

The behavior of water drops deposited on superhydrophobic surfaces under evaporation conditions consists of two states: either the drop sits on the tops of the pillars
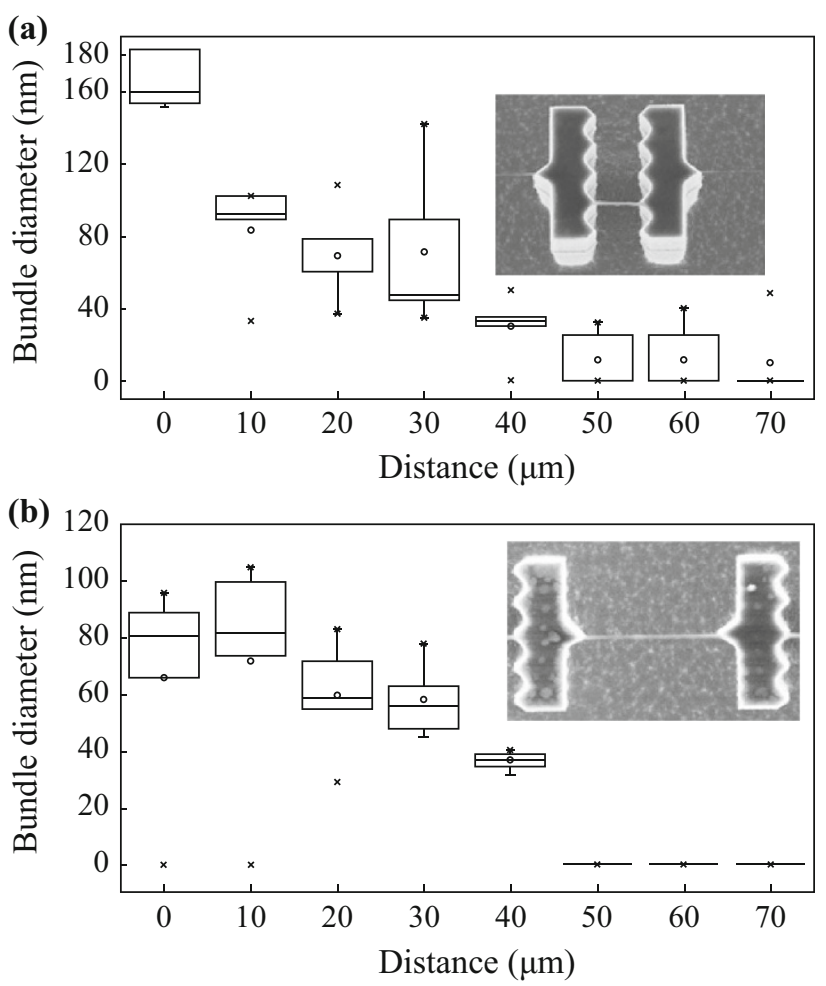

Fig. 6 a The average diameter of the bundle pinned inside the sawshaped pillars in region II as a function of the distance from the DNA stain shown in Fig. 2b. b The average diameter of the bundle pinned outside the saw-shaped pillars as a function of the distance from the stain. A drop volume of $5 \mu \mathrm{L}$ and a DNA concentration of $50 \mathrm{ng} \mu \mathrm{L}^{-1}$ was used

(Cassie state), or it sinks inside the texture (Wenzel state). In the first state, the drop evaporates sitting stably onto the pillars. However, as the drop recedes, a transition from the Cassie state to the Wenzel state may occur [5-15]. This process is schematically displayed in Fig. 2a. After droplet evaporation, a circular stain is usually observed at the bottom of the patterned surface. The typical stain obtained after evaporation of a $5 \mu \mathrm{L}$ DNA solution droplet (150 ng $\mu \mathrm{L}^{-1}$ ) is shown in Fig. 2b. For clarity, three different regions were highlighted on the surface depending on the distance from the stain (Fig. 2b).

In region I, sufficiently distant from the DNA stain, the evaporation dynamics of a droplet in the Cassie State leads to the formation of highly ordered arrays of stretched DNA strands. In this region, DNA strands are strictly suspended on the top of the pillars as previously demonstrated in Ref. [19]. A schematic view and a SEM image of the typical DNA bundles arrangement is shown in Fig. 2c, e, respectively. Conversely, within the DNA stain (region III), the ordered arrangement was usually not present and an unstructured solute deposit was observed instead. The shape and the size of the solute stain may depend on many parameters such as solute concentration, temperature, and pillars shape as recently demonstrated by Dicuangco et al. 
[29]. However, in particular experimental conditions, an ordered arrangement may be observed. In these conditions, DNA may also form bundles pinned at different heights to the body of the pillars suggesting that a tridimensional order can be obtained (Fig. 2d, f) [20].

Moreover, in region I, DNA bundles show a narrow size distribution centered around $20 \mathrm{~nm}$, whereas in region III the average bundle size is about $200 \mathrm{~nm}$. A SEM image of bundles in this region is shown in Fig. 2e. Further details on the bundles behavior in these two limit cases were discussed in Refs. [19] and [20]. However, the bundles behavior in the intermediate region between region I and III (region II), which is endowed with a higher degree of complexity, has not been assessed yet. To address this issue, particular pillar geometry, namely a saw-shaped pillar, was micro-fabricated. The schematic view of the adopted SHS is shown in Fig. 3a. Each pillar is $10 \mu \mathrm{m}$ in side, $14 \mu \mathrm{m}$ pitch, and the distance between the two halves of the same pillar ranges between 4 and $2 \mu \mathrm{m}$ (Fig. 3a). The triangular-shaped protrusions are molded to pin DNA strands both inside and outside the saw-shaped pillars. A SEM image of the SHS is shown in Fig. 3b. The described features make the contemporaneous investigation of multiple length scales possible as clarified in the following.

Figure 4a shows the average diameter of bundles pinned inside the saw-shaped pillars as a function of the distance from the stain in region II. Each value is the average of 15 measurements acquired on three different devices. In the closest proximity to the DNA stain, the bundle diameter is $\sim 160 \mathrm{~nm}$. This value is of the same order of magnitude than that measured within the stain. An exponential decrease of the average bundle diameter down to $\sim 30 \mathrm{~nm}$ can be observed across $170 \mu \mathrm{m}$ from the stain. Since a 6-nm $\mathrm{Cr}$ layer against e-beam strand damage has to be considered, the resulting average bundle diameter at the plateau is $\sim 20 \mathrm{~nm}$, which is consistent with that measured in region III [20].

Figure $4 \mathrm{~b}$ displays the average diameter of the bundles pinned outside the saw-shaped pillars as a function of the distance from the stain in region II. It is clear that the bundles are significantly thinner than those pinned inside the saw-shaped pillars. In the closest proximity to the DNA stain, the bundle size is $\sim 110 \mathrm{~nm}$, which is the same order of magnitude of the diameters distribution that might be observed within the stain. Then, the average bundle diameter decreases monotonously up to reach about $40 \mathrm{~nm}$ at 100 microns far from the stain. From this point, DNA bundles are no longer detected inside the saw-shaped pillars as shown in Fig. 5. This may be due to the fact that bundles pinned outside the saw-shaped pillars are significantly longer and thinner than those pinned inside the pillars and therefore lack the necessary structural support Fig. 6.

Furthermore, we also investigated whether the concentration of the DNA solution plays a significant role in regulating the bundles size. To this purpose, $5 \mu \mathrm{L}$ of $50 \mathrm{ng} \mu \mathrm{L}^{-1}$ genomic DNA solution was deposited on a superhydrophobic surface wetted in the Cassie state. Referencing the bundles pinned outside the saw-shaped pillars, the average bundle diameter dependency on the distance from the stain is shown in Fig. 6a. Like the higher concentrated DNA solution, the bundle diameter is $\sim 160 \mathrm{~nm}$ in the closest proximity to the DNA stain. Then, it decreases exponentially up to reach a plateau of $\sim 15 \mathrm{~nm}$ at $60 \mu \mathrm{m}$ far from the stain. Referencing the bundles pinned outside the saw-shaped pillars (Fig. 6b), the average diameter is $\sim 80 \mathrm{~nm}$ in the closest proximity to the DNA stain and decreases monotonously up to $40 \mathrm{~nm}$, like the more concentrated ones. DNA bundles can no longer be detected at 40 microns far from the stain.

\section{Conclusion}

In this work, we investigated the size distribution and the spatial arrangement of self-assembled DNA bundles formed by droplet evaporation on superhydrophobic surfaces. It was found that both DNA bundle size and its arrangement depended mainly on the droplet evaporation stage. When the droplet was in the Cassie state, ordered array of $\sim 20 \mathrm{~nm}$ DNA filaments was formed. As the evaporation proceeded, a region where the bundle size increased from few tens to few hundreds of nanometers was observed, and the scale of this region depended strongly on the initial DNA concentration. The length of the region is $\sim 170 \mu \mathrm{m}$ at high concentration of $150 \mathrm{ng} \mu \mathrm{L}^{-1}$ and $\sim 40 \mu \mathrm{m}$ at low concentration of $50 \mathrm{ng} \mu \mathrm{L}^{-1}$. Moreover, the bundle size also depended on the distance between adjacent pillars: the larger the distance, the thinner the DNA bundles. In the last phase of the evaporation process, the continuous shrinking of the droplet induced a state transition from Cassie to Wenzel and resulted in a disordered or square-shaped DNA arrangement. Taken together, these results show that the complex evaporation dynamics of a water droplet onto a superhydrophobic surface must be carefully considered when using these surfaces to obtain a self-assembled array of aligned 1D nanostructures. Moreover, the data presented here have the potential to provide a great advantage for those applications where a precise control of the bundle sizes is required.

Acknowledgments The authors are indebted to Mr. Mario Amici for his technical assistance. The experimental data were measured at the LABCEMI (Laboratorio Centralizzato di Microscopia, Ottica ed Elettronica) of the Universitá Cattolica del S.Cuore di Roma (Italy, http://www.rm.unicatt.it/).

Open Access This article is distributed under the terms of the Creative Commons Attribution License which permits any use, distribution, and reproduction in any medium, provided the original author(s) and the source are credited. 


\section{References}

1. B. Su, Y. Wu, L. Jiang, The art of aligning one-dimensional (1D) nanostructures. Chem. Soc. Rev. 41(23), 7832-7856 (2012). doi: $10.1039 / \mathrm{c} 2 \mathrm{cs} 35187 \mathrm{k}$

2. Y. Xia, P. Yang, Y. Sun, Y. Wu, B. Mayers, B. Gates, Y. Yin, F. Kim, H. Yan, One-dimensional nanostructures: synthesis, characterization, and applications. Adv. Mater. 15(5), 353-389 (2003). doi:10.1002/adma.200390087

3. C.S. Torres, Alternative Lithography: Unleashing the Potentials of Nanotechnology (Kluwer Academic/Plenum, New York, 2003)

4. A. Shahmoon, O. Limon, L. Businaro, G. Ciasca, Y. Azugi, A. Gerardino, Z. Zalevsky, Fabrication of an electro-optical Bragg modulator based on plasma dispersion effect in silicon. Microelectron. Eng. 105, 107-112 (2013). doi:10.1016/j.mee.2012.12. 023

5. D. Bartolo, F. Bouamrirene, E. Verneuil, A. Buguin, P. Silberzan, S. Moulinet, Bouncing or sticky droplets: impalement transitions on superhydrophobic micropatterned surfaces. EPL 74(2), 299 (2006). doi:10.1209/ep1/i2005-10522-3

6. M. Reyssat, J.M. Yeomans, D. Quéré, Impalement of fakir drops. EPL 81(2), 26006 (2008). doi:10.1209/0295-5075/81/26006

7. H. Kusumaatmaja, M.L. Blow, A.V.J.M. Dupuis, J.M. Yeomans, The collapse transition on superhydrophobic surfaces. EPL 81(3), 36003 (2008). doi:10.1209/0295-5075/81/36003

8. G. McHale, S. Aqil, N.J. Shirtcliffe, M.I. Newton, H.Y. Erbil, Analysis of droplet evaporation on a superhydrophobic surface. Langmuir 21(24), 11053-11060 (2005). doi:10.1021/la0518795

9. D.H. Kwon, S.J. Lee, Impact and wetting behaviors of impinging microdroplets on superhydrophobic textured surfaces. Appl. Phys. Lett. 100(17), 171601 (2012). doi:10.1063/1.4705296

10. C.W. Extrand, Model for contact angles and hysteresis on rough and ultraphobic surfaces. Langmuir 18(21), 7991-7999 (2002). doi:10.1021/la025769z

11. J. Kijlstra, K. Reihs, A. Klamt, Roughness and topology of ultrahydrophobic surfaces. Colloid Surface A 206(1), 521-529 (2002). doi:10.1016/S0927-7757(02)00089-4

12. Y.C. Jung, B. Bhushan, Contact angle, adhesion and friction properties of micro-and nanopatterned polymers for superhydrophobicity. Nanotechnology 17(19), 4970 (2006). doi:10.1088/ 0957-4484/17/19/033

13. A. Cavalli, P. Bøggild, F. Okkels, Topology optimization of robust superhydrophobic surfaces. Soft Matter 9(7), 2234-2238 (2013). doi:10.1039/c2sm27214h

14. P. Papadopoulos, L. Mammen, X. Deng, D. Vollmer, H.J. Butt, How superhydrophobicity breaks down. Proc. Natl. Acad. Sci. 110(9), 3254-3258 (2013). doi:10.1073/pnas.1218673110

15. Y. Huang, D.K. Sarkar, X.-G. Chen, Fabrication of superhydrophobic surfaces on aluminum alloy via electrodeposition of copper followed by electrochemical modification. Nano-Micro Lett. 3(3), 160-165 (2011). doi:10.3786/nml.v3i3.p160-165

16. B. Su, S. Wang, J. Ma, Y. Wu, X. Chen, Y. Song, L. Jiang, Elaborate positioning of nanowire arrays contributed, by highly adhesive superhydrophobic pillar-structured substrates. Adv. Mater. 24(4), 559-564 (2012). doi:10.1002/adma.201104019

17. B. Su, S. Wang, Y. Wu, X. Chen, Y. Song, L. Jiang, Small molecular nanowire arrays assisted by superhydrophobic pillarstructured surfaces with high adhesion. Adv. Mater. 24(20), 2780-2785 (2012). doi:10.1002/adma.201200294
18. B. Su, Y. Wu, Y. Tang, Y. Chen, W. Cheng, L. Jiang, Freestanding 1D assemblies of plasmonic nanoparticles. Adv. Mater. 25(29), 3968-3972 (2013). doi:10.1002/adma.201301003

19. G. Ciasca, L. Businaro, M. Papi, A. Notargiacomo, M. Chiarpotto, A. De Ninno, V. Palmieri, S. Carta, E. Giovine, A. Gerardino, M. De Spirito, Self-assembling of large ordered DNA arrays using superhydrophobic patterned surfaces. Nanotechnology 24(49), 495302 (2013). doi:10.1088/0957-4484/24/49/495302

20. G. Ciasca, M. Papi, M. Chiarpotto, A. De Ninno, E. Giovine, G. Campi, A. Gerardino, M. De Spirito, L. Businaro, Controlling the cassie-to-wenzel transition: an easy route towards the realization of tridimensional arrays of biological objects. Nano-Micro Lett. 6(3), 280-286 (2014). doi:10.1007/BF03353792

21. G. Campi, G. Ciasca, N. Poccia, A. Ricci, M. Fratini, A. Bianconi, Controlling photoinduced electron transfer via defects selforganization for novel functional macromolecular systems. Curr. Protein Pept. Sci. 14(4), 394 (2014). doi:10.2174/ 1389203715666140327104023

22. F. De Angelis, F. Gentile, F. Mecarini, G. Das, M. Moretti, P. Candeloro, M.L. Coluccio, G. Cojoc, A. Accardo, C. Liberale, R.P. Zaccaria, G. Perozziello, L. Tirinato, A. Toma, G. Cuda, R. Cingolani, E. Di Fabrizio, Breaking the diffusion limit with super-hydrophobic delivery of molecules to plasmonic nanofocusing SERS structures. Nat. Photonics 5, 682-687 (2011). doi:10.1038/nphoton.2011.222

23. F. Gentile, M. Moretti, T. Limongi, A. Falqui, G. Bertoni, A. Scarpellini, S. Santoriello, L. Maragliano, R. Proietti Zaccaria, E. Di Fabrizio, Direct imaging of DNA fibers: the visage of double helix. Nano Lett. 12, 6453-6458 (2012). doi:10.1021/n13039162

24. L. Businaro, O. Limaj, V. Giliberti, M. Ortolani, A. Di Gaspare, G. Grenci, G. Ciasca, A. Gerardino, A. De Ninno, S. Lupi, Midinfrared nanoantenna arrays on silicon and $\mathrm{CaF}_{2}$ substrates for sensing applications. Microelectron. Eng. 97, 197-200 (2012). doi:10.1016/j.mee.2012.02.025

25. L. Di Gaspare, L.G. Ciasca, M. Pea, A. Notargiacomo, Ion and plasma based treatments for enhanced chemical speciation of metals in ferritin. Microelectron. Eng. 124, 86-89 (2014). doi:10. 1016/j.mee.2014.06.003

26. G. Ciasca, L. Businaro, A. De Ninno, A. Cedola, A. Notargiacomo, G. Campi et al., Wet sample confinement by superhydrophobic patterned surfaces for combined X-ray fluorescence and X-ray phase contrast imaging. Microelectron. Eng. 111, 304-309 (2013). doi:10.1016/j.mee.2013.02.020

27. M. Vassalli, F. Sbrana, A. Laurita, M. Papi, N. Bloise, L. Visai, B. Bochicchio, Biological and structural characterization of a naturally inspired material engineered from elastin as a candidate for tissue engineering applications. Langmuir 29(51), 15898-15906 (2013). doi:10.1021/la403311x

28. V. Palmieri, D. Lucchetti, A. Maiorana, M. Papi, G. Maulucci, G. Ciasca, M. Svelto, M. De Spirito, A. Sgambato, Biomechanical investigation of colorectal cancer cells. Appl. Phys. Lett. 105, 123701 (2014). doi:10.1063/1.4896161

29. M. Dicuangco, S. Dash, J.A. Weibel, S.V. Garimella, Effect of superhydrophobic surface morphology on evaporative deposition patterns. Appl. Phys. Lett. 104, 201604 (2014). doi:10.1063/1. 4878322 